Research article

Open Access

\title{
Relationship of patients' age to histopathological features of breast tumours in BRCA1 and BRCA2 and mutation-negative breast cancer families
}

\author{
Hannaleena Eerola ${ }^{1,2}$, Päivi Heikkilä ${ }^{3}$, Anitta Tamminen ${ }^{2}$, Kristiina Aittomäki ${ }^{4}$, Carl Blomqvist ${ }^{1}$ and \\ Heli Nevanlinna ${ }^{2}$
}

\author{
1Department of Oncology, Helsinki University Central Hospital, Finland \\ 2Department of Obstetrics and Gynecology, Helsinki University Central Hospital, Finland \\ ${ }^{3}$ Department of Pathology, Helsinki University Central Hospital, Finland \\ ${ }^{4}$ Department of Clinical Genetics, Helsinki University Central Hospital, Finland
}

Corresponding author: Heli Nevanlinna, heli.nevanlinna@hus.fi

Received: 21 Dec 2004 Revisions requested: 8 Feb 2005 Revisions received: 15 Mar 2005 Accepted: 17 Mar 2005 Published: 21 Apr 2005

Breast Cancer Research 2005, 7:R465-R469 (DOI 10.1186/bcr1025)

This article is online at: http://breast-cancer-research.com/content/7/4/R465

(c) 2005 Eerola et al.; licensee BioMed Central Ltd.

This is an Open Access article distributed under the terms of the Creative Commons Attribution License (http://creativecommons.org/licenses/by/ 2.0), which permits unrestricted use, distribution, and reproduction in any medium, provided the original work is properly cited.

\begin{abstract}
Introduction Our aim was to evaluate the relationship of patients' age to histopathological features of hereditary breast tumours in a series of breast cancer families not selected for age at diagnosis. In sporadic breast cancer, tumours from premenopausal patients have been shown to differ from those of postmenopausal patients, but this phenomenon has been little studied among familial patients.

Methods Representative areas of all available breast cancer tissue specimens $(n=262)$ from $25 B R C A 1,20 B R C A 2$, and 74 non-BRCA1/2 breast cancer families were punched into a tissue microarray. Immunohistochemical staining of oestrogen receptor, progesterone receptor, ERBB2, and p53 as well as the histology and grade of tumours in these three groups of families were studied in different age groups and compared with each other.

Results We found that only breast cancers from young $(<50$ years) $B R C A 1^{+}$patients represent features documented as being typical of BRCA1-associated cancers, such as high tumour grade, negativity for oestrogen and progesterone receptors, and overexpression of p53. Among the BRCA2 families, the opposite was found, with a significantly higher frequency of tumours negative for oestrogen and progesterone receptors among the older patients than among the other groups, but no distinctive tumour characteristics among the younger $B R C A 2$ patients.

Conclusion Tumours of $B R C A 1$ and $B R C A 2$ carriers aged 50 years or more differed significantly from those of younger carriers. This difference may reflect different biological behaviour and pathways of tumour development among the older and the younger BRCA1 and BRCA2 patients, with impact also on prognosis and survival.
\end{abstract}

\section{Introduction}

Distinct pathological features among BRCA1-associated tumours have been found when such tumours are compared with sporadic cancers; these features include high tumour grade, negativity for oestrogen receptor (ER), overexpression of p53, negativity for progesterone receptor (PR), and a higher proportion of medullary and atypical medullary carcinomas [13]. Recently, cDNA expression analyses have suggested a basal epithelial phenotype for $B R C A 1$ tumors [4] and expression of cytokeratins $5 / 6$ have been associated with BRCA1 tumours [5]. Among BRCA2-associated tumours, findings have been inconsistent, and in most cases no significant difference has been found between BRCA2-associated and sporadic cancers $[1,2,6,7]$.

In our previous report [8], we have shown, consistent with earlier studies, that $B R C A 1$-associated cancers were diagnosed younger and were more ER- and progesterone-receptor-negative (PR-), more $\mathrm{p53}^{+}$, and of higher grade than unselected breast tumours or tumours from non-BRCA1/2 breast cancer families. However, in multivariate analysis the independent factors, as compared with non-BRCA1/2 tumours, were age of 
diagnosis, grade, and PR-negativity. BRCA2 cases did not have such distinctive features compared with non-BRCA1/2 cases.

In large studies on sporadic breast cancer tumours, tumours from premenopausal patients have been shown to differ from those of postmenopausal patients [9-11], but this has been little studied among familial patients. In this study, we had an excellent opportunity to study familial cases without age restriction and to evaluate whether the histology and immunohistochemistry differ in the different age groups (according to whether age of diagnosis is below or over 50 years, with age being used as a surrogate for menopause status) among $B R C A 1, B R C A 2$, and non-BRCA1/2 families.

\section{Materials and methods}

Family history of cancer was screened for among breast cancer patients in the Department of Oncology, Helsinki University Central Hospital [12]. Families were collected with a simple criterion of at least three first- or second-degree relatives with breast or ovarian cancer, with no restriction regarding age. All the families were tested for BRCA1 and BRCA2 mutations by mutation analysis of the whole coding sequences and exon/intron boundaries of the genes as described elsewhere $[13,14]$, or were tested for all 18 previously reported Finnish BRCA1 and BRCA2 mutations [13-16]. In this study, as previously described [8], we collected all the available paraffin-wax blocks of the primary breast cancers $(n=262)$ from 119 breast cancer families. Altogether, 51 cancers from the $25 B R C A 1$ families, 59 cancers from the 20 BRCA2 families, and 152 cancers from the 74 non-BRCA1/2 families were obtained.

The patients' median age at diagnosis of the tumours was 44 years for $B R C A 1,47$ years for $B R C A 2$, and 55 years for non$B R C A 1 / 2$. For comparison of tumours from premenopausal and postmenopausal patients, the age of 50 years was chosen as a surrogate for menopause. Among the BRCA1 patients, $34(66.7 \%)$ were diagnosed when they were below 50 years of age (median age 39) and 17 (33.3\%) when they were 50 or more (median age 55); the respective numbers among BRCA2 patients were $35(59.3 \%)$ and 24 (40.7\%) (median ages 39 and 56.5, respectively), and among non-BRCA1/2 patients, $58(38.2 \%)$ and 94 (61.8\%) (median ages 44 and 65 , respectively).

The most representative area of the tumour was punched to produce a hereditary breast cancer tissue microarray including two cores (diameter $0.6 \mathrm{~mm}$ ) from all the original blocks as described elsewhere $[8,17]$. The use of microarray tissue blocks makes it possible to stain all the samples at the same time and in the same conditions. Subgroups of the material are therefore very well comparable, and a highly significant correlation between this kind of multicore system and studying the whole sections of the original blocks has also been shown $[18,19]$.

All the tissue microarray slides were stained with routine methods used for pathological diagnostics with ER, PR, ERBB2, and p53 antibodies in the same laboratory [8]. Briefly, fivemicrometer sections were cut from paraffin-embedded blocks, dewaxed in xylene, and dehydrated in a series of graded alcohols. The sections were pretreated in a microwave oven and incubated with antibody overnight. ER antibody (1:50) and ERBB2 antibody (NCL-CB11, 1:400) were purchased from Novocastra (Newcastle upon Tyne, UK), and PR (1:250) and p53 antibodies (1:100) were from Dako (Copenhagen, Denmark). The evaluation of the staining results was similar to that used in routine diagnostics, and samples were considered positive when $10 \%, 10 \%$, and $20 \%$ of the cells were stained with $E R, P R$, and $p 53$, respectively. Samples having a moderate or intense staining of the entire membrane in more than $10 \%$ of the tumour cells (immunohistochemical scores of $2+$ and $3+)$ were considered to be ERBB2 ${ }^{+}$. Other staining patterns $(0$ and $1+)$ were considered to be negative. We studied the haematoxylin-and-eosin sections of the original blocks to achieve histological diagnosis and grading (all by the same pathologist $(\mathrm{PH})$ ). Statistical analysis was done with SPSS version 8.0 for Windows. We tested the differences in dichotomous variables with a $\chi^{2}$ or Fisher's exact test. All $P$ values are two-tailed.

Permissions for this study were obtained from the ethics committees of the Department of Oncology and the Department of Obstetrics and Gynaecology, Helsinki University Central Hospital, and of the Ministry of Social Affairs and Health in Finland. Blood and tumour samples were used in this study with the informed consent of the probands and of the family members.

\section{Results}

In BRCA1 families, patients whose cancer was diagnosed when they were under 50 years of age differed significantly from those diagnosed at 50 years or older in the presence of grade 3 tumours (84.4\% vs $47.1 \%, P=0.009)$, ER-negativity (83.3\% vs $25 \%, P=0,001)$, and p53-positivity $(50.0 \%$ vs $7.7 \%, P=0.014$ ) (Table 1). All of the five cancers with medullary histology were also detected in patients under 50 years old. Patients who were $B R C A 1^{+}$and were under 50 years old at diagnosis differed significantly in all of these factors from familial non-BRCA1/2 patients (proportion of grade 3 tumours, $84.4 \%$ vs $17.3 \%$, respectively, $P \leq 0.0005$; of ERnegativity, $83.3 \%$ vs $29.3 \%, P \leq 0.0005$; of PR-negativity, $90.3 \%$ vs $31.0 \%, P \leq 0.0005$; and of p53-positivity, $50.0 \%$ vs $25.9 \%, P=0.024)$. However, patients from $B R C A 1$ families diagnosed at age 50 years or older differed significantly only for grade from the non-BRCA1/2 patients in the same age group $(47.1 \%$ vs $23.3, P=0.044)$. 
Features of breast tumours according to breast cancer patient's age (years) at diagnosis

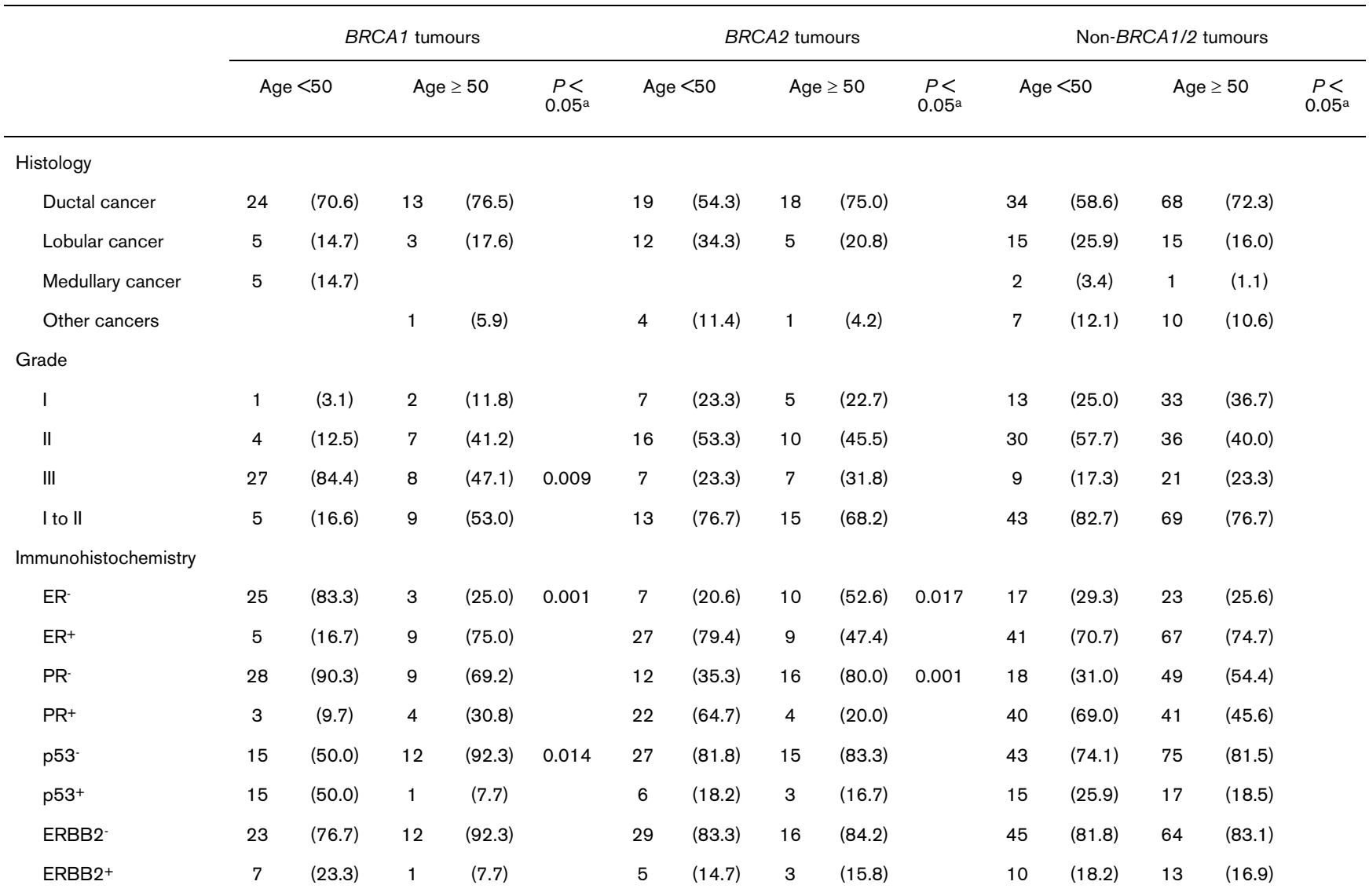

Values are no. (frequency \%). aComparison by age group. There were no significant differences according to patients' age in the non-BRCA1/2 tumours (rightmost column). ER, oestrogen receptor; ERBB2, ERBB2 oncoprotein; PR, progesterone receptor.

In BRCA2 families, tumours of patients diagnosed at less than 50 years of age differed significantly from those of the older patients for ER-negativity (20.6\% vs $52.6 \%$, respectively, $P=$ $0.017)$ and PR-receptor negativity $(35.3 \%$ vs $80.0 \%, P=$ 0.001 ) (Table 1). In contrast to BRCA1 tumours, the BRCA2 tumours diagnosed in patients 50 years or older were more often ER $(52.6 \%$ vs $25.6 \%, P=0.02)$ and PR $(80.0 \%$ vs $54.4 \%, P=0.036)$ than non-BRCA1/2 cancers among the same age group. Tumours of patients diagnosed at less than 50 years of age were very similar to non-BRCA1/2 tumours in the same age group (Table 1).

Pathological features of non-BRCA1/2 tumours did not vary significantly between the two age groups.

\section{Discussion}

In this study, we have evaluated whether tumour histology and immunohistochemistry are influenced by age of onset (menopause status) among families with $B R C A 1, B R C A 2$, or non$B R C A 1 / 2$ tumours. Most of the earlier studies of the characteristics of tumours in $B R C A 1$ and $B R C A 2$ carriers have been based on young patients only. Because there was no age restriction in our selection criterion, we had an excellent opportunity to study patients within the whole age distribution.

In BRCA1 families, tumours from patients diagnosed at over 50 years of age were surprisingly different from those in $B R C A 1$ carriers diagnosed at under 50 years. Only tumours from the younger patients exhibited the distinctive characteristics that have been found to be typical of BRCA1 tumours, with higher grade, negativity for ER and $P R$, and positivity for p53 distinguishing them from familial non-BRCA1/2 tumours. However, tumours from the older patients in BRCA1 families differed significantly only in grade from tumours in non$B R C A 1 / 2$ patients. There were only five cases among this older group of patients, for which the BRCA1 mutation status was unknown. If these patients are excluded from the analysis, the observed frequencies remain ; therefore those do not account for the result.

Previously, Vaziri and colleagues [20] have reported that the tumour immunophenotype of BRCA1-carriers is influenced by the age of diagnosis. As a control group, those authors used age-matched breast cancer patients unselected for family history, whereas in our study we included familial non-BRCA1/2 
cancer cases. Vaziri and colleagues observed no differences in ER or PR staining of tumours between BRCA1 carriers diagnosed at 50 years or older and controls with sporadic cancers [20]. Foulkes and colleagues [21] also recently reported that the proportion of $\mathrm{ER}^{+}$tumours increased with patients' age among the BRCA1 patients included in their study (diagnosed at less than 65 years of age), although they found a strong relationship between BRCA1 carrier status and ERnegativity of tumours in the age group 55 to 65 years. Vaziri and colleagues also studied the expression of markers Ki-67, Cyclin D1, p53, and ERBB2. None of these markers differed significantly in the patients 50 years or older between BRCA1associated cancers and control cancers, although tumours from the younger BRCA1 age group presented less frequent $E R, P R$, and cyclin D1 staining and more frequent Ki-67 and B-catenin staining than those from control cancers. p53 expression did not differ in their study in different age groups, nor was p53 more frequently overexpressed among young $B R C A 1$ patients than in controls.

We did not find the BRCA2-associated tumours to differ significantly from familial non-BRCA1/2 tumours among the younger age group. However, tumours of BRCA2 carrier patients diagnosed at 50 years or older had more distinctive features, and were more ER- and PR-, than tumours of younger patients or tumours of the same age group of $B R C A 1$ patients or non-BRCA1/2 patients.

The specific features of BRCA1-associated tumours among the younger age group, and lack of such features among the $B R C A 2$-associated tumours, are consistent with the overall characteristics reported previously among BRCA1 and $B R C A 2$ patients $[1,2]$. Such features characterise to a large extent the BRCA1 and BRCA2 tumours overall, as a large majority (63\% in this study) of all breast tumours in the $B R C A 1$ and BRCA2 families are diagnosed before patients reach 50 years of age.

However, among both BRCA1 and BRCA2 families, tumours from older patients form subgroups that are distinctly different from those of the younger patients. Tumours from the older $B R C A 1$ patients resemble more those among the mutationnegative families, or sporadic tumours. The highest incidence rates and relative breast cancer risk among $B R C A 1$ carriers are seen before age 50 [22], and some tumours from older $B R C A 1$ mutation carriers could also be 'sporadic' cancers. However, the breast tumours from older BRCA1 patients also differed from mutation-negative ones by their higher grade. Furthermore, tumours from the older BRCA2 carriers exhibited distinctly different characteristics from the younger ones or from BRCA1 carrier tumours and mutation-negative ones, suggesting a strong impact of the germline mutation on tumour development among the older patients. It is interesting that the BRCA1 and BRCA2 tumours appear to be opposites with respect to their characteristics in the age groups of younger and older patients.

There are now many models and computer programs to test the probability of $B R C A 1$ or BRCA2 mutations [23-28]. We have also documented previously that efficient predictors for $B R C A 1$ and BRCA2 mutations are early age of breast cancer onset and number of ovarian cancer cases in the family [27]. Simple family history criteria of the strongest predictors (onset of breast cancer under age 40 and presence of ovarian cancer) for a mutation may also provide a rough estimate of a high likelihood of carrying a mutation [27].

However, it would be useful if, besides family history, histopathological markers could also be used to distinguish patients and families likely to carry a $B R C A 1 / 2$ germline mutation from mutation-negative families and breast cancer patients in general. The use of morphologic and immunohistochemical data has been previously suggested to provide a helpful and cost-effective tool for predicting BRCA1 mutation among young breast cancer patients $[2,29,30]$. The findings here provide further information specifically with respect to older BRCA1 and BRCA2 patients and warrant further studies for evaluating the probability of mutation by combining information on family history and tumour characteristics in the various age groups.

\section{Conclusion}

These findings may reflect different biological behaviour and pathway of tumour development among the older and the younger $B R C A 1$ and $B R C A 2$ patients, with impact also on prognosis and survival. So far, results on survival among $B R C A 1$ and BRCA2 patients have been inconclusive or contradictory, and large meta-analyses specifically according to age groups could shed further light on this. Finally, in the context of genetic counselling, specific tumour characteristics may help evaluate the possibility of a $B R C A 1$ or $B R C A 2$ mutation and the need for mutation testing in a family with a history of breast cancer. It appears crucial, however, to consider such features specifically with respect to the age of the patients.

\section{Competing interests}

The author(s) declare that they have no competing interests.

\section{Authors' contributions}

HE drafted the manuscript, participated in the design of the study and data collection, and performed the statistical analysis. PH carried out the immunohistochemistry. AT carried out the molecular genetic studies. KA participated in patient collection and did the genetic counselling of the patients and participated in drafting the manuscript. CB participated in the design of the study and drafting of the manuscript. HN participated in the design of the study, data collection, and drafting of the manuscript. All authors read and approved the final manuscript. 


\section{Acknowledgements}

We wish to thank the families that participated in this study, Minna Merikivi for her help in sample collection, and the Finnish Cancer Registry for cancer diagnosis and identification numbers for archival material of the pathology laboratories. Grants: Academy of Finland, Clinical Research Fund of Helsinki University Central Hospital, Finnish Breast Cancer Group, Finnish-Norwegian Medical Foundation, The Finnish Cancer Society, and Sigrid Juselius Foundation.

\section{References}

1. Phillips $\mathrm{K}$, Andrulis IL, Goodwin PJ: Breast carcinomas arising in carriers of mutations in BRCA1 or BRCA2: Are they prognostically different? J Clin Oncol 1999, 17:3653-3663.

2. Lakhani SR, van de Vijver MJ, Jacquemier J, Anderson TJ, Osin PP, McGuffog L, Easton D, Consortium BCL: The pathology of familial breast cancer: Predictive value of immunohistochemical markers estrogen receptor, progesterone receptor, HER-2, and p53 in patients with mutations in BRCA1 and BRCA2. $J$ Clin Oncol 2002, 20:2310-2318.

3. Malone KE, Daling JR, Ostrander EA: BRCA1 and medullary breast cancer. JAMA 1998, 280:1227.

4. Sorlie T, Tibshirani R, Parker J, Hastie T, Marron JS, Nobel A, Deng $\mathrm{S}$, Johnsen H, Pesich R, Geisler S, et al:: Repeated observation of breast tumour subtypes in independent gene expression data sets. Proc Natl Acad Sci 2003, 100:8418-8423.

5. Foulkes W, Stefansson IM, Chappuis PO, Begin LR, Goffin JR, Wong N, Trudel M, Akslen LA: Germline BRCA1 mutations and basal epithelial phenotype in breast cancer. J Natl Cancer Inst 2003, 95:1482-1485.

6. Noguchi S, Kasugai T, Miki Y, Fukutomi T, Emi M, Nomizu T: Clinicopathologic analysis of BRCA1 - or BRCA2 associated hereditary breast carcinoma in Japanese Women. Cancer 1999, 85:2200-22205.

7. Vahteristo $P$, Syrjäkoski K, Eerola H, Kainu T, Holli K, Blomqvist $\mathrm{C}$, Kallioniemi O, Nevanlinna H: Response: re: population-based study of BRCA1 and BRCA2 mutations in 1035 unselected Finnish breast cancer patients. J Natl Cancer Inst 2001, 93:152-154.

8. Eerola $\mathrm{H}$, Heikkilä $\mathrm{P}$, Tamminen A, Aittomäki $\mathrm{K}$, Blomqvist $\mathrm{C}$, Nevanlinna $\mathrm{H}$ : Histopathological features of breast tumours in BRCA1, BRCA2, and in mutation negative breast cancer families. Breast Cancer Research 2004 in press.

9. Nixon AJ, Neuberg D, Hayes DF, Gelman R, Connolly JL, Schnitt S, Abner A, Recht A, Vicini F, Harris JR: Relationship of patient age to pathologic features of the tumor and prognosis for patients with stage I or II breast cancer. J Clin Oncol 1994, 12:888-894.

10. Talley LI, Grizzle WE, Waterbror JW, Brown D, Weiss H, Frost AR: Hormonen receptors and proliferation in breast carcinomas of equivalent histologic grades in pre- and postmenopaussal women. Int J Cancer 2002, 98:118-127.

11. Anderson WF, Chu KC, Chang S, Sherman ME: Comparison of age-spesific insidence rate patterns for different histopathologic types of breast carcinoma. Cancer Epidemiol Biomarkers Prev 2004, 13:1128-1135.

12. Eerola $H$, Blomqvist $C$, Pukkala $E$, Pyrhönen $S$, Nevanlinna $H$ : Familial breast cancer in southern Finland: how prevalent are breast cancer families and can we trust the family history reported by patients? Eur J Cancer 2000, 36:1143-1148.

13. Vehmanen P, Friedman LS, Eerola H, Sarantaus L, Pyrhönen S, Ponder BAJ, Muhonen T, Nevanlinna H: A low proportion of BRCA2 mutations in Finnish Breast cancer families. Am J Hum Genet 1997, 60:1050-1058.

14. Vehmanen $\mathrm{P}$, Friedman LS, Eerola $\mathrm{H}$, McClure M, Ward B, Sarantaus L, Kainu T, Syrjäkoski K, Pyrhönen S, Kallioniemi O, et al.: Low proportion of BRCA1 and BRCA2 mutations in Finnish breast cancer families: evidence for additional susceptibility genes. Hum Mol Genet 1997, 6:2309-2315.

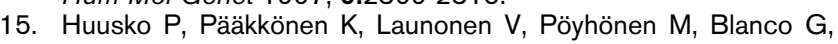
Kauppila A, Puistola U, Kiviniemi H, Kujala M, Leisti J, et al.: Evidence of founder mutations in Finnish BRCA1 and BRCA2 families. Am J Hum Genet 1998, 62:1544-1548.

16. Sarantaus L, Huusko $P$, Eerola $H$, Launonen V, Vehmanen $P$, Rapakko K, Gillanders E, Syrjäkoski K, Kainu T, Vahteristo P, et al.: Multiple founder effects and geographical clustering of BRCA1 and BRCA2 families in Finland. Eur J Hum Genet 2000, 8:757-763.

17. Vahteristo $P$, Bartkowa J, Eerola H, Syrjäkoski K, Ojala S, Kilpivaara $\mathrm{O}$, Tamminen A, Kononen J, Aittomäki K, Heikkilä P, et al: A CHK2 genetic variant contributing to a substantial fraction of familial breast cancer. Am J Hum Genet 2002, 71:432-438.

18. Vaziri AJ, Krumroy LM, Elson P, Budd GT, Darlington G, Myles J, Tubbs RR, Casey G: Breast tumour immunophenotype of BRCA1-mutation carriers is influenced by age at diagnosis. Clin Cancer Res 2001, 7:1937-1945.

19. Antoniou AC, Pharoah P, Narod S, Risch H, Eyfjord JE, Hopper JL, Loman N, Olsson H, Johansson OT, Borg A, et al.: Average risks of breast and ovarian cancer associated with mutations in BRCA1 or BRCA2 detected in case series unselected for family history: a combined analysis of 22 studies. Am J Hum Genet 2003, 72:1117-1130.

20. Foulkes W, Metcalfe K, Sun P, Hanna WM, Lynch H, Ghadirian P, Tung $\mathrm{N}$, Olopade O, Weber BL, McLennan J, et al:: Estrogen reseptor status in BRCA1- and BRCA2-related breast cancer: The influence of age, grade, and histological type. Clin Cancer Res 2004, 10:2029-2034.

21. Kononen J, Bubendorf $L$, Kallioniemi $A$, Bärlund $M$, Schraml $P$, Leighton S, Torhorst J, Mihatch MJ, Sauter G, Kallioniemi O: Tissue microarrays for high-throughput molecular profiling of tumor specimens. Nature Med 1998, 4:844-847.

22. Gillet CE, Springall RJ, Barnes DM, Hanby AM: Multiple tissue core arrays in histopathology research: a validation study. $J$ Pathol 2000, 192:549-553.

23. Shattuck-Eidens D, Oliphant A, McClure M, McBride C, Gupte J, Rubano T, Pruss D, Tavtigian SV, Teng DH, Adey N, et al.: BRCA1 sequence analysis in women at high risk for susceptibility mutations. Risk factor analysis and implications for genetic testing. JAMA 1997, 278:1242-1250.

24. Couch FJ, DeShano ML, Blackwood MA, Calzone K, Stopfer J, Campeau L, Ganguly A, Rebbeck T, Weber BL: BRCA1 mutations in women attending clinics that evaluate the risk of breast cancer. N Engl J Med 1997, 336:1409-1415.

25. Chan-Claude J, Dong J, Schmidt S, Shayeghi M, Komitowski D, Becher $\mathrm{H}$, Stratton MR, Royer-Pokora B: Using gene carrier probability to select high risk families for identifying germline mutations in breast cancer susceptibility genes. J Med Genet 1998, 35:116-121.

26. Parmigiani G, Berry D, Aguilar O: Determining carrier probabilities for breast cancer-susceptibility genes BRCA1 and BRCA2. Am J Hum Genet 1998, 62:145-158.

27. Vahteristo $P$, Eerola $H$, Tamminen $A$, Blomqvist $C$, Nevanlinna $H$ : A probability model for predicting BRCA1 and BRCA2 mutations in breast and breast-ovarian cancer families. Brit $J$ Cancer 2001, 84:704-708.

28. Gilpin CA, Carson N, Hunter AGW: A preliminary validation of a family history assessment form to select women at risk for breast or ovarian cancer for referral to a genetic center. Clin Genet 2000, 58:299-308.

29. Lidereau R, Eisinger $F$, Champème $M-H$, Noguès $C$, Bièche I, Birnbaum D, Pallud C, Jacquemier J, Sobol H: Major Improvement in the efficacy of BRCA1 mutation screening using morphoclinical features of breast cancer. Cancer Res 2000, 60:1 206-1210.

30. Eisinger $F$, Noguès $C$, Guinebretiere J, Peyrat J, Bardou V, Noguchi $T$, Vennin $P$, Sauvan R, Lidereau R, Birnbaum D, et al:: Novel indications for BRCA1 screening using individual clinical and morphological features. Int J Cancer 1999, 84:263-267. 